\title{
2 ESTROGEN RECEPTOR $\alpha(E R \alpha)$ EXPRESSION IN CARTILAGE IS IMPORTANT FOR THE AMELIORATING EFFECTS OF ESTROGEN ON SYNOVITIS, BUT NOT JOINT DESTRUCTION.
}

Cecilia Engdahl', Anna E Börjesson', Annica Andersson', Alexandra Stubelius ${ }^{1}$ Andree Krust ${ }^{2}$, Pierre Chambon², Ulrika Islander ${ }^{1}$, Claes Ohlsson ${ }^{1}$, Hans Carlsten ${ }^{1}$ and Marie K Lagerquist ${ }^{1}{ }^{1}$ Centre for Bone and Arthritis Research, Inst. of Medicine, Gothenburg University, Sweden; ${ }^{2}$ GBMC, IIIkirch, France

10.1136/annrheumdis-2011-201237.2

Objective Estrogen ameliorates incidence and progression of rheumatoid arthritis.. The aim of this study was to investigate the importance of cartilage-specific ER $\alpha$ expression for the ameliorating effects of estrogen on arthritis.

Methods Mice with total (total $E R \alpha^{-/}$) or cartilage-specific $\left(\mathrm{Col} 2 \alpha 1-\mathrm{ER} \alpha^{-/-}\right)$inactivation of $\mathrm{ER} \alpha$ and wild-type mice were ovariectomised, treated with estradiol $(0.83 \mu \mathrm{g} / \mathrm{day})$ or placebo and induced with antigen-induced-arthritis (AIA). At termination, knees were collected for histology, synovial cells and splenic T cells were investigated using flow cytometry and T cell proliferation and cytokine production was examined.

Results Estrogen protected against AIA-induced synovitis and joint destruction in wild-type mice and this was associated with decreased frequencies of inflammatory cells (neutrophils, monocytes/macrophages and $\mathrm{T}$ cells) in synovial tissue and decreased systemic T cell proliferation. No estrogenic effect was seen in total $E R \alpha$ deficient mice. In cartilage-specific $E R \alpha$ inactivated mice, estrogen protected against joint destruction and decreased systemic $T$ cell proliferation to a similar extent as in wild-type mice. In contrast, estrogen did not ameliorate synovitis in mice lacking ER $\alpha$ in cartilage.

Conclusions Estrogen ameliorates both synovitis and joint destruction in AIA via $\mathrm{ER} \alpha$ and this decreased severity in arthritis is associated with a decrease in synovial inflammatory cells and decreased T cell proliferation. Interestingly, ER $\alpha$ expression in cartilage is required for the ameliorating estrogenic effects on synovitis but not joint destruction, suggesting different target cells and mechanisms for the estrogenic protection of synovial inflammation and joint destruction. 